\title{
Persistence of Chlorpropham (CIPC) in the Concrete Flooring of Potato Stores
}

\author{
Leisa Douglas ${ }^{1}$ (D) - Gillian MacKinnon ${ }^{1}$. \\ Gordon Cook $^{1}$ - Harry Duncan ${ }^{2}$ - Adrian Briddon ${ }^{3}$. \\ Steven Seamark ${ }^{3}$
}

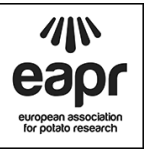

Received: 8 February 2018 / Accepted: 6 June 2018 /

Published online: 7 July 2018

(C) The Author(s) 2018

\begin{abstract}
The loss of the sprout suppressant, chlorpropham (CIPC), to the fabric of potato stores is currently of concern due to the risk of potential cross contamination of other crops subsequently housed in these stores. HPLC UV/VIS and GCMS methods were successfully employed to detect CIPC in the concrete flooring of research and commercial potato stores with histories of between 1 and 26 years of use. The concentrations in identical research stores, with different numbers of applications, were in the range $0.58-5.7$ and 3.4-112 $\mu \mathrm{g} \mathrm{g}^{-1}$, suggesting the magnitude of contamination was influenced by the number of applications. Commercial store A, with a history of 18 seasons of applications (estimate of total CIPC applied $2040 \mathrm{~kg}$ ), had concentrations varying between 6 and $48 \mu \mathrm{g} \mathrm{g}^{-1}$ in the top three centimetres, with more than $92 \%$ within the top centimetre. In contrast, commercial store B, with a history of less than five seasons of applications (estimate of total CIPC applied $319 \mathrm{~kg}$ ), had concentrations varying between 0.58 and $304 \mu \mathrm{g} \mathrm{g}^{-1}$ in the top four centimetres, with less than $47 \%$ within the top centimetre. The difference in depth distributions between A and B may be due to the structural integrity of the concrete, which was much poorer in B. CIPC was persistent in all stores irrespective of the total quantities of CIPC applied and date of the final application.
\end{abstract}

Keywords Chlorpropham $\cdot$ Concrete $\cdot$ Persistence $\cdot$ Storage

Leisa Douglas

1.douglas.2@research.gla.ac.uk; leisaantoinettedouglas@yahoo.co.uk

1 Scottish Universities Environmental Research Centre, Rankine Avenue, G75 0QF East Kilbride, UK

2 School of Chemistry, University of Glasgow, Joseph Black Building, Edinburgh G12 8QQ, UK

3 AHDB Sutton Bridge Crop Storage Research, Lincolnshire PE12 9YD, UK 


\section{Introduction}

Globally, potatoes are an essential food crop (Gómez-Castillo et al. 2013) and their long-term storage allows year-round availability (Foukaraki et al. 2016). Proper management of potato stores is critical to maintain crop quality throughout the storage period (AHDB-Potatoes 2011). The long-term storage of potato tubers can be achieved by controlling temperature and humidity in stores (Cools et al. 2014; Foukaraki et al. 2016) and by the application of sprout suppressants (Cools et al. 2014). The sprout suppressant chlorpropham (CIPC, isopropyl $\mathrm{N}$-(3-chlorophenyl) carbamate) is most commonly used (Blenkinsop et al. 2002) and is typically applied in the UK as a hot fog (spherical particles of about 5- $\mu \mathrm{m}$ diameter) on stored potatoes (Gouseti et al. 2015). This process is efficient in controlling sprouting through deposition of solid CIPC on the potatoes (Gouseti et al. 2015); however, it is also deposited on the fabric of the store (Smith et al. 2013), particularly on the walls and flooring (Boyd and Duncan 1986). Previously, there was no requirement for monitoring CIPC residue levels on the fabric of potato stores. However, this has become more relevant due to the emergence of cross contamination issues of other crops, such as grains, that are housed in buildings previously used as potato stores (AHDB-Potatoes 2012; Red Tractor Assurance ${ }^{\mathrm{a}}$ 2014). Cross contamination of non-potato crops has been recognised for some time, as stipulated on chlorpropham product labels; however, due to developing legislations, there is now an increased awareness of this issue. The latest changes to chlorpropham product labels, confirmed by the Chemical Regulation Division of the Health and Safety Executive (HSE), ensure compliance with the Maximum Residue Level (MRL) of $10 \mathrm{mg} \mathrm{kg}{ }^{-1}$ (10 ppm) for potatoes set by the European Commission (EC Regulation 1107/2009). Chlorpropham may also be used as a herbicide in the production of a range of food and ornamental crops (Gard and Rudd 1953; Cessna and Benoit 1992; Murkute et al. 2013; BCPC 2018) and MRLs are set at the limit of quantification for salad crops such as lettuce $\left(0.05 \mathrm{mg} \mathrm{kg}^{-1}\right)$, onions $\left(0.05 \mathrm{mg} \mathrm{kg}^{-1}\right)$, teas and coffee $\left(0.05 \mathrm{mg} \mathrm{kg}^{-1}\right)$ and herbs and edible flowers ( $0.02 \mathrm{mg} \mathrm{kg}^{-1}$ ) (HSE 2017). The MRL for cereals (grains) is also assigned at the limit of quantification which is $0.01 \mathrm{mg} \mathrm{kg}^{-1}$ (European Food Safety Authority 2011; HSE 2017).

Assessment of CIPC in the fabric of a building previously used for storing potatoes is therefore now essential, prior to storage of other commodities, as the presence of CIPC may render it unfit for storage (Red Tractor Assurance ${ }^{b}$ 2014). Also, the maintenance of accurate records pertaining to buildings with a history of CIPC use is crucial for preventing cross contamination of crops that have no clearance for CIPC (AHDB-Potatoes 2012), which is a recommendation that the Potato Industry CIPC Stewardship Group has supported since its establishment in 2008 (Paul et al. 2016).

The stability of CIPC in soil and aquatic environments is well known (Smith and Bucher 2012). However, its persistence in building materials has not been fully explored. Therefore, information pertaining to the possible route(s) and degree of cross contamination is scant. It has been postulated that cross contamination of grains or seed potatoes may be caused by residual CIPC in the concrete fabric of the stores (Douglas et al. 2018) but evidence for the accumulation of residues in concrete is very limited. Boyd and Duncan (1986) employed gas chromatography flame ionisation detection (GC-FID) to determine CIPC and found concentrations ranging from 130 to $290 \mathrm{mg} \mathrm{kg}^{-1}$ in the concrete walls and 2050 to $9470 \mathrm{mg} \mathrm{kg}^{-1}$ in the flooring. In a recent study, where CIPC concentrations were quantified in a research potato store, Douglas et al. (2018) showed that approximately $90 \%$ was retained in the first centimetre of the concrete flooring, with detection up 
to a depth of $4 \mathrm{~cm}$. The persistence and heterogeneity of CIPC distribution in the concrete flooring of the assessed potato stores was not considered by either Boyd and Duncan (1986) or Douglas et al. (2018). Therefore, there is a need to explore its distribution and persistence following several years of use, to better understand the current situation. In this study, the distribution of CIPC in the concrete flooring of two research and two commercial potato stores was investigated, with the heterogeneity of distribution being assessed in one of the commercial stores. The chosen examples included buildings that previously were used for storage of potatoes and were treated with CIPC between 1 and 100 times.

\section{Material and Methods}

\section{Chemicals and Reagents}

Isopropyl N-(3-chlorophenyl) carbamate (CIPC, 98\% purity) was purchased from Sigma-Aldrich (Dorset, UK). HPLC-grade water was produced in a Millipore Elix® 5 water purification system (Molsheim, France) and HPLC-grade acetone and acetonitrile were purchased from Fisher Scientific (Loughborough, UK). A stock solution of $1000 \mu \mathrm{g} \mathrm{mL}^{-1}$ of CIPC was prepared in acetonitrile and stored at $4{ }^{\circ} \mathrm{C}$. Calibration standard solutions $\left(0.01-1.0 \mu \mathrm{g} \mathrm{mL}^{-1}\right)$ were prepared from the stock solution.

\section{Store Designs and Histories}

Commercial stores A and B were bulk potato stores with dimensions (length $\times$ width) of $36.5 \mathrm{~m} \times 18.2 \mathrm{~m}$ and $18.3 \mathrm{~m} \times 13.7 \mathrm{~m}$, respectively. The capacities of stores A and B were 2000 and $200 \mathrm{t}$, respectively. Potatoes were stored at a depth of 4.5 and $1.8 \mathrm{~m}$ in stores A and B, respectively. The point of CIPC entry into store A was under the fan, at the back of the store, via a main duct continuous with all underground lateral ducts $(7.2 \mathrm{~m} \times 0.38 \mathrm{~m} \times 0.3 \mathrm{~m}$, length $\times$ width $\times$ depth; Fig. 1a). CIPC was applied into the main duct using a Superfog machine (Superfog 2018). An axial fan (1.2-m diameter) mounted $1.5 \mathrm{~m}$ above floor level was used for ventilation, but was not used during CIPC applications.

The point of CIPC entry into store B was via galvanised ducts 2 and 4 and occasionally duct 6 (Fig. 2). CIPC was applied as a fog, directly into the ducts, using a Swingfog machine (Swingtec 2018).

CIPC applications ceased to stores A and B with final applications in 2000 and 1990, respectively. This allows worst-case estimates for the total amount of CIPC applied to each store to be calculated, based on the maximum application rate of $63.75 \mathrm{~g} \mathrm{CIPC}^{-1}$ during the lifetime of the store and the metric tonne capacity of the store. Estimates, using the store histories of the commercial stores, indicated the total amount of CIPC applied to store A was $2040 \mathrm{~kg}\left(63.75 \mathrm{~g} \mathrm{t}^{-1} \times 16\right.$ years $\left.\times 2000 \mathrm{t}\right)$ and to store $\mathrm{B}$ was $319 \mathrm{~kg}\left(63.75 \mathrm{~g} \mathrm{t}^{-1} \times 25\right.$ years $\left.\times 200 \mathrm{t}\right)$. Research stores 1 and 2 were identical in design with dimensions $3.5 \mathrm{~m} \times 5.13 \mathrm{~m}$ (length $\times$ width) and a capacity of $12 \mathrm{t}$ of potatoes, in boxes. CIPC was applied as a fog and recirculated by a plenum ventilation system. It was introduced via a duct in the back door (Fig. 3). The total amounts applied at the time of this study were at least $8 \mathrm{~kg}$ for research store 1 and a single application of $0.108 \mathrm{~kg}$ for research store 2 . 

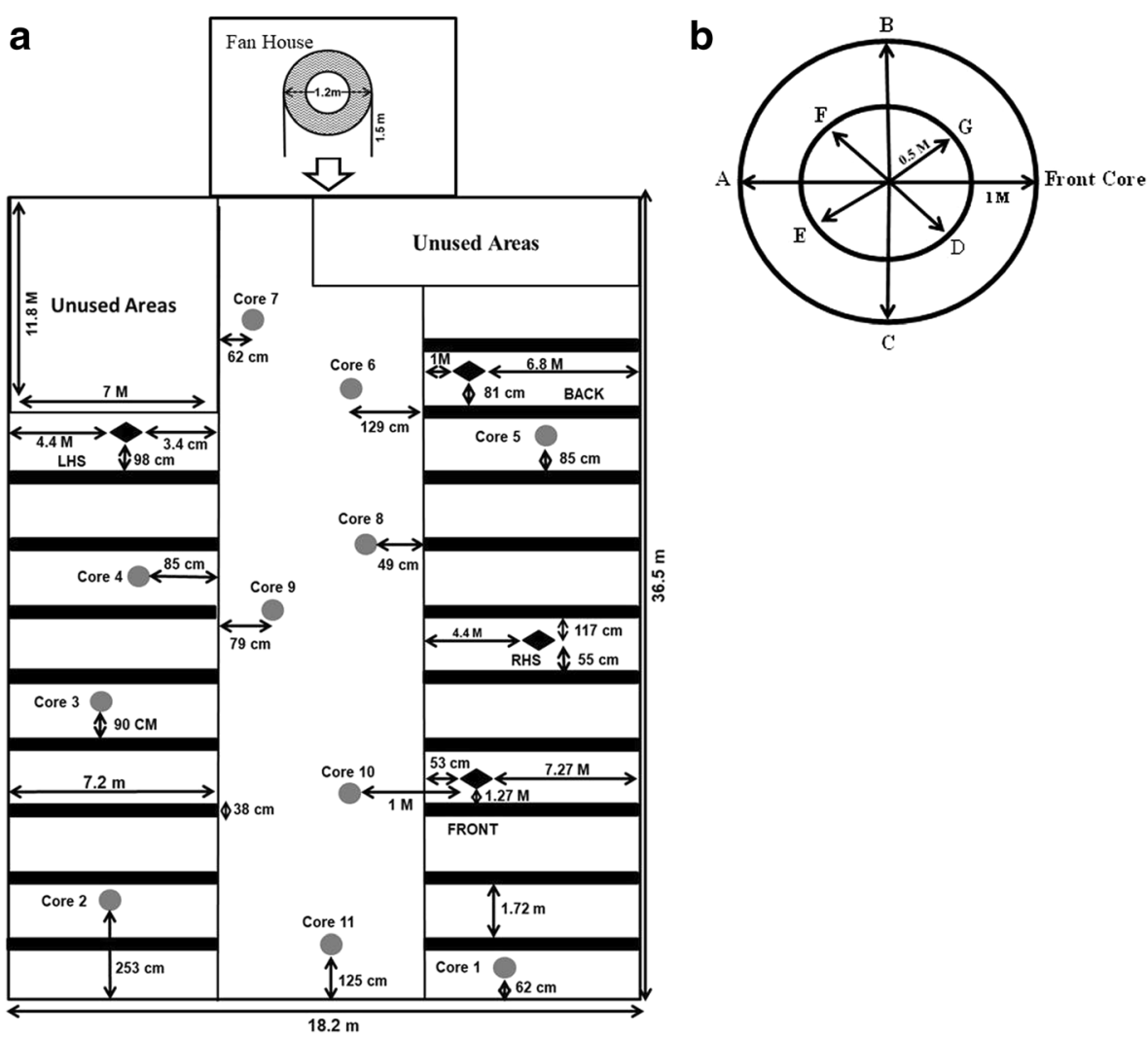

Fig. 1 a Floor plan for commercial store A, indicating positions of cores. Black rectangles represent the lateral duct outlets that were relevant in the sampling pattern. LHS and RHS represent the left-hand side and right-hand side of the store respectively. The main duct is represented by the arrow inside the fan house which points in the direction of the store. b Diagrammatic representation of the systematic sampling pattern showing the inner and outer circles around central core number 10 in commercial store A

\section{Sample Collection and Preparation for CIPC Analysis}

Cores (4-cm diameter) were collected from the concrete floors of two identical research stores and two commercial stores. The length of the core recovered depended on the ease of penetration of the drill into the concrete. Cores were sectioned for depth distribution studies using the cutting procedure and sample processing methodology detailed by Douglas et al. (2018). Three cores were collected at measured distances near the plenum (store ventilation system), the front of the store and the back of the store (point of CIPC entry) for both research stores. Four cores were collected from commercial store A at measured distances from evenly distributed lateral ducts (points of CIPC entry) (Fig. 1a). These preliminary cores were collected on the left-hand side, right-hand side, front and back of the store. Eighteen additional cores, using random and systematic sampling patterns, were also collected from commercial store A to assess the heterogeneity of CIPC distribution in the flooring (Fig. 1a). The first 1-cm layers of the additional cores were analysed. Eight cores were collected in proximity to the front core using a systematic sampling pattern (Fig. 1b). The sampling pattern had a central core (core number 10) which was positioned $1 \mathrm{~m}$ from the front core. Cores A, 


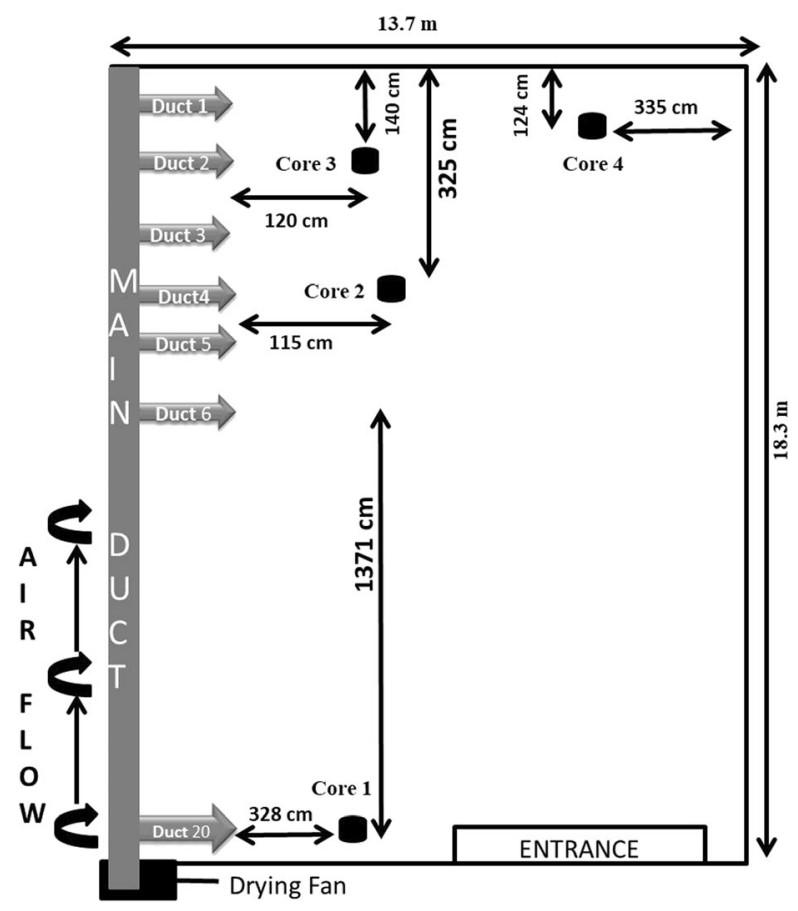

Fig. 2 Floor plan of commercial store B showing position of cores (black discs)

$\mathrm{B}, \mathrm{C}$ and the front core, were positioned $1 \mathrm{~m}$ from the central core in a cross pattern and formed an outer circle around the central core. Cores D, E, F and G were positioned at 0.5-m distances in a diagonal pattern from the central core, forming an inner circle pattern around the central core. Four cores were collected from commercial store B at measured distances from the ducts (points of CIPC entry) (Fig. 2). Three of these cores crumbled and were not used for the depth distribution study. The portions of crumbled concrete for the respective cores were processed together. The depth distribution of CIPC in the stores was determined by calculating the concentration $\left(\mu g^{-1}\right)$ in each
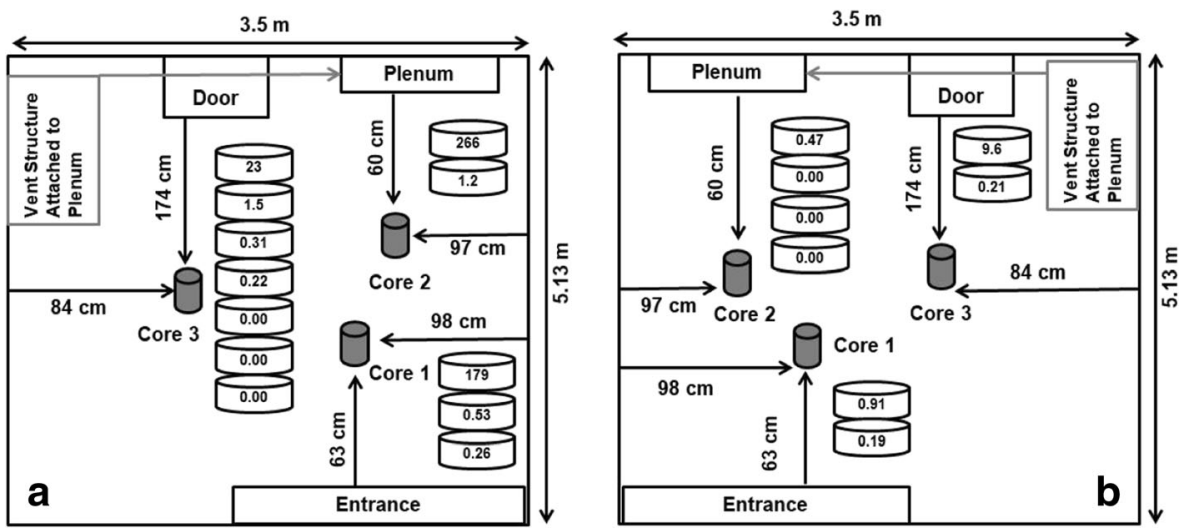

Fig. 3 Diagrammatic representation of a research store 1 (several applications) and b research store 2 (single application), indicating positions of cores (grey cylinders) and CIPC concentrations $\left(\mu \mathrm{g} \mathrm{g}^{-1}\right)$ in each $1-\mathrm{cm}$ layer (disc). Depth of CIPC distribution depicted by descending discs 
layer of the respective cores. The lateral distribution was calculated as the total concentration in individual cores $\left(\mu \mathrm{g} \mathrm{g}^{-1}\right)$. This was done using the same cores from the depth distribution study for the respective stores. The mass of CIPC in each layer of each core was calculated then the mass in each layer summed and converted to a total concentration $\left(\mu \mathrm{g} \mathrm{g}^{-1}\right)$ by dividing by the total weight of the uncrushed core.

\section{CIPC Analysis in Concrete}

The concrete samples were extracted and analysed by HPLC UV/VIS and GCMS as detailed in Douglas et al. (2018) with the exception that the HPLC UV/VIS column was washed with acetonitrile (100\%) for 30 to $40 \mathrm{~min}$ between analyses. The extended wash time ensured that there was no carryover caused by co-extracted residues in the commercial concrete.

\section{Results and Discussion}

\section{Preliminary Evaluation of the HPLC UV/VIS Method for Detecting CIPC in a Commercial Store}

A concrete sample collected from commercial store A was crushed, split into three subsamples and extracted to test the reproducibility of the extraction and HPLC UV/ VIS method. A spiked blank concrete matrix at a nominal CIPC concentration of $4.0 \mu \mathrm{g} \mathrm{g}^{-1}$ and an extraction blank containing only acetonitrile was also taken through the extraction and analysis processes. The similar CIPC concentrations obtained for the sample from commercial store A (mean $=9.7 \mu \mathrm{g} \mathrm{g}^{-1} \pm 3.49 \% \mathrm{RSD}, n=3$ ) suggested that the procedure for processing the cores (crushing and mixing) prior to analysis was sufficient for sample homogenisation and that the method was precise. The CIPC spiked blank concrete matrix gave a recovery of $97 \%$ and the extraction blank gave no peak signal for CIPC, confirming the robustness of the processing, extraction and analytical procedures. The method developed by Douglas et al. (2018) was validated for accuracy, precision and linearity using reconstituted concrete blocks (Bradstone, UK). Preliminary assessment of the method was successful in quantifying CIPC in research stores. It was noted that the appearance of the concrete samples from the commercial stores was different from the concrete used in the method development (Douglas et al. 2018). This was evident in the physical characteristics of the crushed samples and in the HPLC UV/VIS analysis. Although an extended analysis time was required to remove co-extracted residues from the HPLC column, the HPLC UV/VIS method was also successful in quantifying CIPC in the commercial store samples.

\section{Confirmation of CIPC in Concrete Using GCMS}

The presence of CIPC in the industrial concrete samples was confirmed using GCMS. The GCMS spectral patterns were consistent with the expected CIPC spectrum (213/ $215 \mathrm{~m} / \mathrm{z}$ : parent ion; 153/154 m/z: m-chlorophenyl isocyanate; 171/173 m/z: free acid formed from isopropyl residue; $127 / 129 \mathrm{~m} / \mathrm{z}$ : chloroaniline) as outlined on the NIST database: (http://webbook.nist.gov/cgi/cbook.cgi?Name=chlorpropham \&Units= $\mathrm{SI \& cMS}=$ on\#Mass-Spec). 


\section{Depth Distribution of CIPC in Potato Stores}

Cores collected from research stores 1 and 2 (three in each store) and the preliminary cores collected from commercial store A (four cores) and commercial store B (one core) were used in the depth distribution study. The research store data are shown in Fig. 3 while the commercial store data are given in Table 1. Three of the four cores collected from commercial store B crumbled during coring and could not be layered; however, one intact core was obtained and was used to determine the depth distribution.

The majority of CIPC (90-100\%) was found in the first 1-cm layer of each core from research stores 1 and 2 (Fig. 3) and commercial store A (Table 1), with a trend of decreasing concentrations in the subsequent layers. CIPC was shown to penetrate the concrete flooring of the stores to a depth of between 3 and $4 \mathrm{~cm}$. In commercial store A, it penetrated the concrete to a depth of at least $3 \mathrm{~cm}$ and has persisted for the 16 years since the last application. In the research stores, higher concentrations were obtained for store 1 which had many applications (at least $8 \mathrm{~kg}$ CIPC), relative to store 2, with only one application $(0.108 \mathrm{~kg}$ CIPC). CIPC had penetrated the concrete flooring to a depth of 4 and $2 \mathrm{~cm}$ in research stores 1 and 2, respectively. In both stores, more than $90 \%$ residual CIPC was retained in the first centimetre. The differences in the total concentration and depth distribution can be related to the number of applications and the degree of contamination (Fig. 3).

In commercial store $\mathrm{B}, 47 \%$ of the CIPC remained in the first 1-cm layer of the core with 35,15 and $4 \%$ in the $2 \mathrm{nd}, 3 \mathrm{rd}$ and 4 th depth increments, respectively, quite different from the depth distributions in the other three stores (Table 1 and Fig. 3). The low percentage of CIPC in the first 1-cm layer and the degree of penetration into the lower layers may be explained in terms of the structural integrity of the concrete, as the concrete from this store seemed porous and of poorer quality relative to the other stores, and this is reflected in the fact that three of the four cores crumbled during sampling. This suggests that CIPC penetration in the concrete flooring is influenced by the composition of the concrete and the efficiency with which the CIPC is applied to the actual potatoes (as opposed to the store in general). The high concentration found in commercial store B, 25 years since the last application, also confirmed its persistence in concrete.

Table 1 CIPC concentration $\left(\mu \mathrm{g} \mathrm{g}^{-1}, n=1\right)$ and percentages of total in parentheses in each layer of each core from commercial stores A and B

\begin{tabular}{|c|c|c|c|c|}
\hline & \multicolumn{3}{|c|}{ Concentration of CIPC in each layer in the cores $\left(\mu \mathrm{g} \mathrm{g}^{-1}\right)$} & \multirow[b]{2}{*}{4 th $\mathrm{cm}$} \\
\hline & $1 \mathrm{st} \mathrm{cm}$ & $2 \mathrm{nd} \mathrm{cm}$ & $3 \mathrm{rd} \mathrm{cm}$ & \\
\hline \multicolumn{5}{|c|}{ Position of cores in commercial store A } \\
\hline Back & $23(91)$ & $2.0(8)$ & $0.20(0.8)$ & NS \\
\hline Right-hand side & $17(98)$ & $0.38(2)$ & NS & NS \\
\hline Left-hand side & $29(98)$ & $0.66(2)$ & NS & NS \\
\hline Front & $104(94)$ & $4.5(4)$ & $1.8(2)$ & NS \\
\hline \multicolumn{5}{|c|}{ Position of core in commercial store B } \\
\hline Duct 2: core 3 & $472(47)$ & $352(35)$ & $150(15)$ & $36(4)$ \\
\hline
\end{tabular}


NS-not sampled.

\section{Lateral Distribution of CIPC in Potato Stores}

\section{The Magnitude of CIPC Contamination and the Influence of the Number of Applications}

The magnitude of CIPC contamination in the concrete flooring of the research stores increased with the total quantities applied and vice versa in the commercial stores. Using identical research stores, research store 1, which had 23 years of CIPC applications, had higher total concentrations $\left(3.4-112 \mu \mathrm{g} \mathrm{g}^{-1}\right)$ relative to research store $2\left(0.58-5.6 \mu \mathrm{g} \mathrm{g}^{-1}\right)$, which only had one application. This is almost certainly due to the higher quantities applied to store 1 (at least $8 \mathrm{~kg}$ ) which resulted in the accumulation and retention of residues in the concrete. In contrast, commercial store A, with a history of 18 seasons (estimate $2040 \mathrm{~kg}$ of applied CIPC), had concentrations varying between 6 and $48 \mu \mathrm{g} \mathrm{g}^{-1}$ in the top three centimetres; while commercial store B with a history of five seasons (estimate $319 \mathrm{~kg}$ of applied CIPC) had concentrations varying between 0.57 and $304 \mu \mathrm{g} \mathrm{g}^{-1}$ in the top four centimetres (Table 2). CIPC was persistent in all stores irrespective of the total quantities of CIPC applied.

\section{The Degree of Contamination Near the Point of CIPC Entry}

The concentrations of CIPC in concrete floors at the point of entry into a store are likely to be greater than those in areas that are remote from the point of entry. This applies particularly when there is no circulatory system to evenly distribute the CIPC, as was the case in commercial store B. CIPC entered this store (as a fog) via above-ground ducts with no additional circulatory system to effect an even distribution. As such, it was observed that the highest concentrations were found in the concrete cores that were near the entry ducts (core 3, $216 \mu \mathrm{g} \mathrm{g}^{-1}$ and core 2, $304 \mu \mathrm{g} \mathrm{g}^{-1}$; Fig. 2 and Table 2). The third highest concentration in commercial store B was observed at the back righthand side of the store, aligned with the point of CIPC entry into the store but remote from it. This core had a much lower concentration (core 4, $10 \mu \mathrm{g} \mathrm{g}^{-1}$; Fig. 2 and

Table 2 Total CIPC concentration $\left(\mu \mathrm{g} \mathrm{g}^{-1}\right)$ in each core for commercial stores A and B

\begin{tabular}{lc}
\hline Position of cores & CIPC concentration $\left(\mu \mathrm{g} \mathrm{g}^{-1}\right)$ \\
\hline Commercial store A & 6.7 \\
Back & 7.8 \\
Right-hand side & 18 \\
Left-hand side & 48 \\
Front & \\
Commercial store B & 0.57 \\
Front: core 1 & 304 \\
Duct 4: core 2 & 216 \\
Duct 2: core 3 & 10 \\
Duct 2: core 4 &
\end{tabular}


Table 2) relative to the core that was nearest to the point of entry (core 3, $216 \mu \mathrm{g} \mathrm{g}^{-1}$; Fig. 2 and Table 2). The lowest concentration was observed at the front of the store (core $1,0.57 \mu \mathrm{g} \mathrm{g}^{-1}$; Fig. 2 and Table 2) which was remote from all entry ducts. The contamination of concrete at the front of the store may be due to fog emanating from the top of the bulk pile and filling the store headspace.

\section{The Effect of Circulation on the CIPC Distribution}

CIPC in commercial store A was applied as a fog via lateral ducts that were evenly positioned throughout the store. After application, a fan located at the back of the store was used to blow air into the main duct. The lowest CIPC concentration was observed from the core at the back of the store $\left(6.7 \mu \mathrm{g} \mathrm{g}^{-1}\right)$ while the highest was observed at the front core $\left(48 \mu \mathrm{g} \mathrm{g}^{-1}\right)$ (Table 2). The CIPC concentrations also differed for the cores that were collected on the left-hand side $\left(18 \mu \mathrm{g} \mathrm{g}^{-1}\right)$ and right-hand side $\left(7.8 \mu \mathrm{g} \mathrm{g}^{-1}\right)$ of the store (Table 2). The inconsistent CIPC distribution may be attributed to (i) the uneven covering of the floor by the potato bulk or (ii) the lateral duct system used to distribute the applied CIPC.

A plenum system used in the research stores illustrates the effect of circulation on distribution. The order of CIPC distribution can be represented in terms of the concentration levels in the three identical cores collected from both stores. The distributions from highest to lowest concentrations were in the order: plenum (112 $\left.\mu \mathrm{g} \mathrm{g}^{-1}\right)$, front $\left(63 \mu \mathrm{g} \mathrm{g}^{-1}\right)$ and back $\left(3.4 \mu \mathrm{g} \mathrm{g}^{-1}\right)$ for research store 1 and back $\left(5.6 \mu \mathrm{g} \mathrm{g}^{-1}\right)$, front $\left(0.58 \mu \mathrm{g} \mathrm{g}^{-1}\right)$ and plenum $\left(0.11 \mu \mathrm{g} \mathrm{g} \mathrm{g}^{-1}\right)$ for research store 2 (Table 3). The concentration trend observed for research store 2 was expected, based on the plenum circulatory system, while for store 1 , the observed trend was unexpected. On further investigation into the history of store 1, it was found that CIPC was not circulated into the store prior to the installation of the plenum system. The trend observed for store 1 may be complicated by accumulation of CIPC in the concrete prior to the use of the plenum system.

\section{Heterogeneity of CIPC Distribution in a Commercial Store}

Commercial store A was selected for further distribution studies because the concrete flooring was easily cored and produced intact cores that could be sectioned. The first $1 \mathrm{~cm}$ of each core was selected for analysis because greater than $90 \%$ of the CIPC was found in this layer, based on preliminary analysis of four cores (Fig. 4). The first 1-cm layers of other cores, collected in a systematic pattern and random pattern (Fig. 4), were

Table 3 Total CIPC concentration $\left(\mu \mathrm{g} \mathrm{g}^{-1}\right)$ in each core for research stores 1 and 2

\begin{tabular}{lcc}
\hline & CIPC concentrations $\left(\mu \mathrm{g} \mathrm{g}^{-1}\right)$ in research stores \\
\hline Position in stores & 1 & 2 \\
Front: core 1 & 63 & 0.58 \\
Plenum: core 2 & 112 & 0.11 \\
Back: core 3 & 3.4 & 5.6 \\
\hline
\end{tabular}




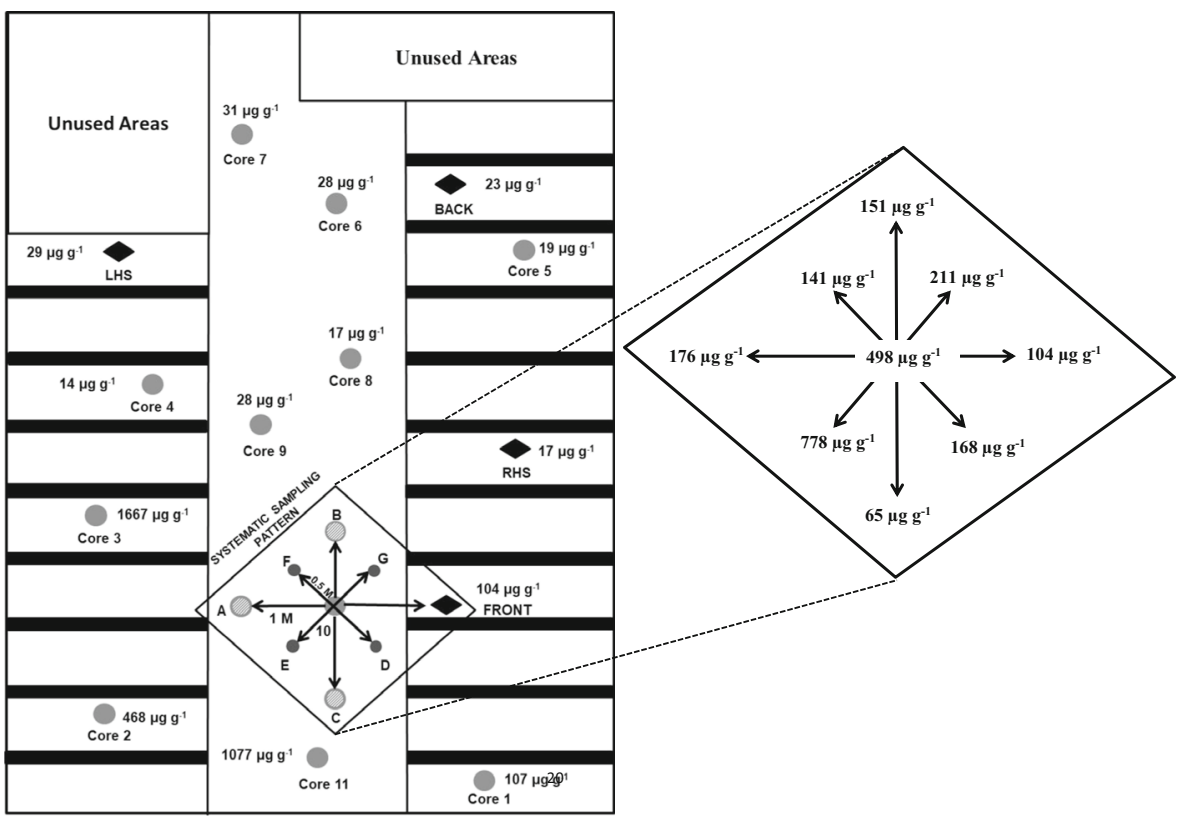

Fig. 4 Floor plan for commercial store A, indicating positions of cores and respective CIPC concentrations in the first 1-cm layer of the cores. Black rectangles represent the lateral duct outlets that were relevant in the sampling pattern. LHS and RHS represent the left-hand side and right-hand side of the store respectively

assessed. The heterogeneity of distribution in commercial store A was determined using the concentration in the top centimetre of the cores.

Systematic sampling from around a central front core with a concentration of $498 \mu \mathrm{g} \mathrm{g}^{-1}$ produced values that ranged from 65 to $778 \mu \mathrm{g} \mathrm{g}^{-1}$. Random sampling highlighted that the distribution was lowest $\left(14-31 \mu^{-1} \mathrm{~g}^{-1}\right)$ at the back of the store and highest at the front $\left(104-1667 \mu \mathrm{g} \mathrm{g}^{-1}\right)$. There was one point of CIPC entry at the back of the store where the fogger duct terminates. The CIPC introduced into the lateral duct system is then distributed into the store. It is therefore conceivable that the concentrations at the front of the store would be higher relative to the back. The information provided from the heterogeneity study confirms the expected distribution pattern.

\section{Conclusion}

The total distribution of CIPC in the concrete flooring of the four stores that were investigated indicates that (i) the magnitude of CIPC remaining in concrete floors is influenced by the total quantities of CIPC applied and on the physical nature of the concrete; (ii) concrete areas closest to the point of CIPC entry are more likely to have higher concentrations and generally, the degree of contamination is variable within a store; (iii) CIPC concentrations in concrete are influenced by the type of circulatory system installed in the store; (iv) CIPC is persistent in concrete irrespective of the number of seasons over which it is applied to the stored potatoes; and (v) CIPC can persist in the concrete flooring for decades after the last application. This is evident in a commercial store where CIPC was detected up to a depth of $4 \mathrm{~cm}, 25$ years since the 
last application. The persistent nature of CIPC highlights the risk of possible cross contamination of other commodities housed in potato stores with a history of CIPC use. The information provided in this study will be used to inform decontamination strategies, as it provides an indication of the depth of concrete that would require removal or treatment to allow the stores to be re-used.

Acknowledgements The authors would like to thank Darran Bill, Dale Green and Graeme Stroud (AHDBPotatoes: Sutton Bridge Crop Storage Research) for assistance with coring; Dr. Hugh Flowers, Michael Beglan and Gangi Reddy Ubbara (University of Glasgow) for technical assistance; Jim Imlach (Scottish Universities Environmental Research Centre, SUERC) for assistance with sectioning of the cores and Alice Sin (Research Executive, AHDB-Potatoes) for useful discussions.

Funding Information This research was funded by the Agriculture and Horticulture Development Board (AHDB) - Potatoes (Project Code: 115R485).

\section{Compliance with Ethical Standards}

Conflict of Interest The authors declare that they have no conflict of interest.

Open Access This article is distributed under the terms of the Creative Commons Attribution 4.0 International License (http://creativecommons.org/licenses/by/4.0/), which permits unrestricted use, distribution, and reproduction in any medium, provided you give appropriate credit to the original author(s) and the source, provide a link to the Creative Commons license, and indicate if changes were made.

\section{References}

AHDB-Potatoes (2011) Store managers guide. Available online at: https://potatoes.ahdb.org. uk/sites/default/files/publication_upload/Store\%20Managers\%20Guide\%20Updated\%2011.05.12.pdf [Accessed: 01 Mar 2017]

AHDB-Potatoes (2012) Avoid risks of CIPC cross contamination. Available online at: https://potatoes.ahdb. org.uk/news/avoid-risks-cipc-cross-contamination [Accessed: 01 Mar 2017]

Blenkinsop RW, Copp LJ, Yada RY, Marangoni AG (2002) Effect of chlorpropham (CIPC) on carbohydrate metabolism of potato tubers during storage. Food Res Int 35:651-655

Boyd WD, Duncan HJ (1986) Studies on potato sprout suppressants. 7. Headspace and residue analysis of chlorpropham in a commercial box potato store. Potato Res 29:217-223

British Crop Production Council The UK Pesticide Guide 2018. Products approved for use in agriculture, amenity, forestry, pest control and horticulture. ISBN: 978-1-9998966-0-7 Ed. M.A. Lainsbury

Cessna A, Benoit DL (1992) Weed control and herbicide residues in onion following use of chlorpropham and cyanazine. Pestic Sci 35:355-362

Cools K, Almar MDC, Terry LA (2014) Controlling sprouting in potato tubers using ultraviolet-C irradiance. Postharvest Biol Technol 98:106-114

Douglas L, MacKinnon G, Cook G, Duncan H, Briddon A, Seamark S (2018) Determination of chlorpropham (CIPC) residues, in the concrete flooring of potato stores, using quantitative (HPLC UV/VIS) and qualitative (GCMS) methods. Chemosphere 195:119-124

European Food Safety Authority (EFSA) (2011) The 2009 European Union report on pesticide residues in food. European Food Safety Authority Journal 9(11):2430

Foukaraki SG, Cools K, Chope GA, Terry LA (2016) Impact of ethylene and 1-MCP on sprouting and sugar accumulation in stored potatoes. Postharvest Biol Technol 114:95-103

Gard LN, Rudd NG (1953) Herbicides determination, isopropyl N-(3-Chlorophenyl) carbamate (CIPC) in soil and crops. J Agric Food Chem 1(9):630-632

Gómez-castillo D, Cruz E, Iguaz A, Arroqui C, Vi'rseda P (2013) Effects of essential oils on sprout suppression and quality of potato cultivars. Postharvest Biol Technol 82:15-21 
Gouseti O, Briddon A, Saunders S, Stroud G, Fryer PJ, Cunnington A (2015) CIPC vapour for efficient sprout control at low applications levels. Postharvest Biol Technol 110:239-246

Health and Safety Executive (2017) MRL Annex Description. Available online at: https://secure.pesticides. gov.uk/MRLs/search.asp [Accessed: 20 Dec 2017]

Murkute AA, Singh B, Gopal J (2013) Residue analysis and bulb sprouting in CIPC treated onion. Indian J Hort 70(4):575-579

Paul V, Ezekiel R, Pandey R (2016) Sprout suppression on potatoes: need to look beyond CIPC for more effective and safer alternatives. J Food Sci Technol 53(1):1-18

Red Tractor Assurance ${ }^{\mathrm{a}}$ (2014) Fresh produce. Available online at: http://assurance.redtractor.org. uk/contentfiles/Farmers-5954.pdf [Accessed: 01 Mar 2017]

Red Tractor Assurance ${ }^{\mathrm{b}}$ (2014) Combinable crops and sugar beet. Available online at: http://assurance. redtractor.org.uk/contentfiles/Farmers-5891.pdf. [Accessed: 01 Mar 2017]

Regulation (EC) No. 1107/2009 of the European Parliament and of the Council concerning the placing of plant protection products on the market. Official Journal of the European Union. 24.11.2009

Smith MJ, Bucher G (2012) Tools to study the degradation and loss of the N-phenyl carbamate chlorprophamA comprehensive review. Environ Int 49:38-50

Smith MJ, Müller S, Sander W, Bucher G (2013) Mechanisms of the thermal decay of chlorpropham. J Hazard Mater 246-247:154-162

Superfog (2018) Superfog efficient economical sprout control in potato storage. Available online at: http://superfog.co.uk/ [Accessed: 03 May 2018]

Swingtec (2018) Swingfog® High Performace Fog Generators. Available online at: https://www.swingtec. de/thermal-fogging-machines/swingfog-product-overview.htm [Accessed: 03 May 2018] 